\title{
Adaptive Filtering by Non-Invasive Vital Signals Monitoring and Diseases Diagnosis
}

\author{
Omar Abdallah ${ }^{1,2}$ and Armin Bolz ${ }^{1}$ \\ ${ }^{1}$ Institute for Biomedical Engineering, Karlsruhe Institute of Technology \\ ${ }^{2}$ Biomedatronik, Karlsruhe
}

Germany

\section{Introduction}

The reliability, reproducibility and accuracy of in-vivo measurements are of great importance and have to be thoroughly studied and to a great extend achieved. Reproducibility problems may result from the electronic components of the applied devices and the variability of measured variables as well as noise sources. The inaccuracy is caused by the approximation in the calculations or the used methods and by diverse sources of errors resulting from the subject under considerations and its surroundings. In sensible measurement like blood components, the positioning of the measuring sensor as well as the variation in the applied pressure and the characteristics of contact area between sensor and skin have a great effect on the accuracy and reproducibility of the measurements. The ambient noise like high frequency and line frequency $(50$ or $60 \mathrm{~Hz}$ ) noise can be filtered by the detected biosignals like Photoplethysmogram (PPG) using the conventional analog or digital filters without a great effort. The motion artifact of the subject caused by him as well as by physical motion of body parts or by the surrounding has a varying frequency which may have the same range of the signal frequency. It is difficult to filter noise from these signals, and errors resulting from filtering can distort them. Usually physicians are misled by these noisy signals and the analysis can go wrong. An adaptive filter is essential by biosignal and bio-image processing for noise cancellation without destroying or manipulating the valuable detected information.

Biomedical signals such as photoplethysmogram (PPG) (Figure 1), electrocardiogram (ECG), electroencephalogram (EEG), electromyogram (EMG) and impedance cardiogram (ICG) are very important in the diagnosis of different pathological variations. By the detection of these bio-signals as well as by the further derived parameters like oxygen saturation by pulse oximeter, the motion artifact is a great challenge, which may lead to erroneous results or even no results can be delivered [Lee].

The effectiveness of ECG monitors can be significantly impaired by motion artifact, which can cause misdiagnoses, lead to inappropriate treatment decisions or trigger false alarms. However, it is difficult to separate the noise from bio-signal due to its frequency spectrum overlapping that of the ECG. A portable ECG recorder using accelerometer based on motion artifact removal technique will be a great help for patients for tele-homecare or ambulatory ECG monitoring. 


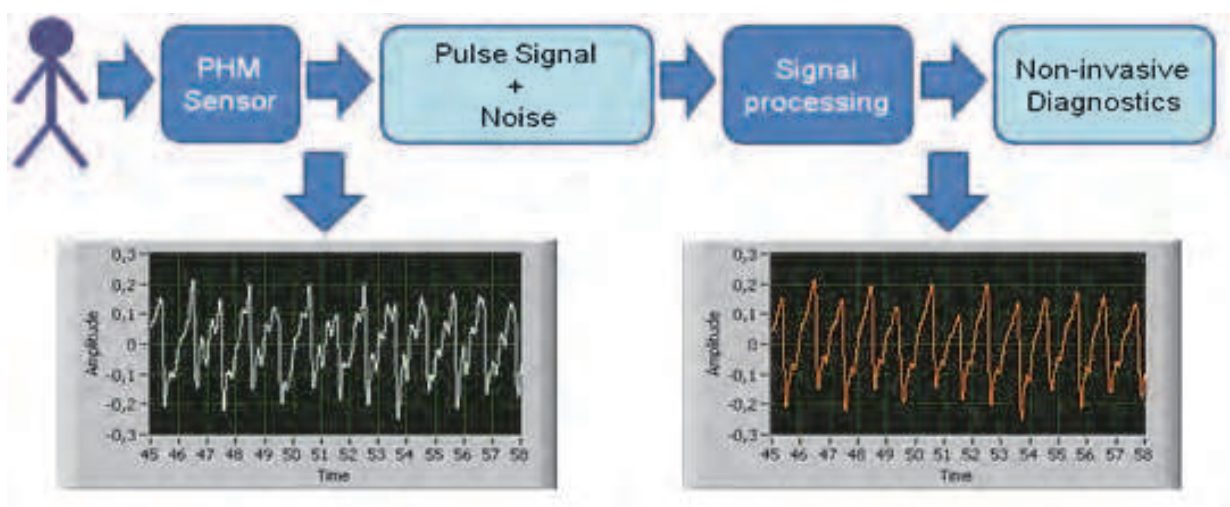

Fig. 1. Signal detection and processing by noninvasive diagnosis

A maternal electrocardiogram (mECG) and abdominal noise in abdominal maternal recordings (especially by cardiotocography) can be orders of magnitude stronger than the fetal electrocardiogram (fECG) signal. An adaptive filter using frequency-domain or timedomain electrocardiogram features can be applied by the automatically extraction of a beatto-beat fECG from mECG using surface electrodes placed on the maternal abdomen [Rik] [Prasad]. This will allow early diagnosis and monitoring treatment of certain fetal cardiac disorders.

By non-contact ECG monitoring, cardiac activity and movements (that may be seen in part in cardioballistogram CBG) may cause also disturbance to the detected signals, which can be eliminated by applying an appropriate adaptive filter

High-quality EEG recording is crucial for diagnosis of different pathological variations. EEG has biological artifacts and external artifacts. Biological artifacts can be EMG-, EOG(Electrooculograph) CBG or ECG-signal [Rasheed]. These artifacts appear as noise in the recorded EEG signal individually or in a combined manner. These noise sources increase the difficulty in analyzing the EEG and to obtaining clinical information. For this reason, it is necessary to design specific filters to decrease such artifacts in EEG records. EEG quality in the MR scanner is compromised by artifacts caused by interaction between the subject, EEG electrode assemblies, and the scanner's magnetic fields [Rasheed2009]. The three most significant causes of EEG artifacts in the scanner are the large movements in the static field like swallowing; the cardioballistogram and blood flow effects in the field associated with the subject's pulse; and the changing fields applied during fMRI image acquisition. Pulse artifact is potentially a significant problem as it is normally large amplitude, widespread on the scalp, and continuous. Using a cascade of adaptive filters based on a least mean squares (LMS) algorithm can eliminate the undesired signals or interferences.

\section{Photoplethysmogram}

The photoplethysmogram (PPG) waveform comprises a pulsatile physiological waveform superimposed on a slowly varying baseline with various lower frequency components. The pulsatile one is attributed to cardiac synchronous changes in the blood volume with each heart beat, and the second is attributed to respiration, sympathetic nervous system activity and thermoregulation. Figure 2 shows a typical PPG signal without motion artifact. The 
PPG technology has been used in a wide range of commercially available medical devices for measuring oxygen saturation, blood pressure and arterial stiffness, cardiac output, assessing autonomic function and detecting peripheral vascular diseases. Although the origins of the components of the PPG signal are not fully understood, there is no doubt that they can provide valuable information about the cardiovascular system and autonomic nervous system. Hence, there is a great interest in the technique in recent years, driven by the demand for low cost, very compact size, simple and portable technology for the primary care and community based clinical settings, non-invasive technology without side effects or risks as well as online monitoring capability and the advancement of computer-based pulse wave analysis techniques and diagnosis [Allen, Abicht]. A computer aided analysis tool for the hemodynamic diagnosis using PPG can be very helpful in clinical applications. Automatic assessment of the reliability of reference heart rates from patient vital-signs monitors incorporating both ECG and PPG based pulse measurements has been proposed by $\mathrm{Yu}$ et al. They expressed reliability as a quality index for each reference heart rate. The physiological waveforms were assessed using a support vector machine classifier and the independent computation of heart rate made by an adaptive peak identification technique that filtered out motion-induced noise [Allen].

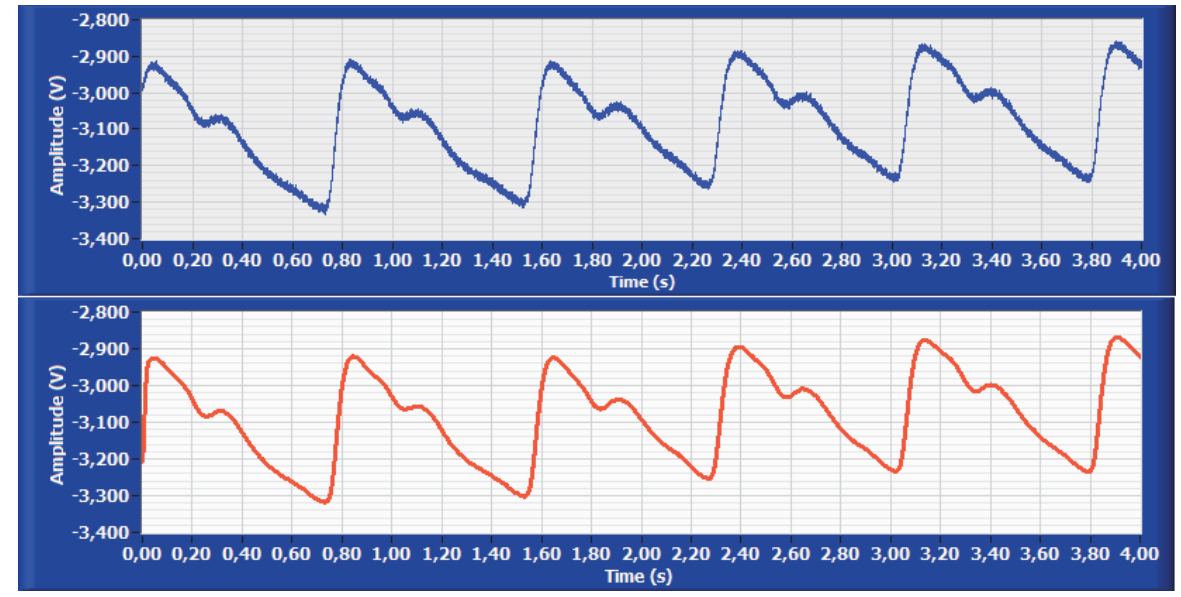

Fig. 2. Photoplethysmogram PPG; top: detected raw signal, bottom: filtered signal

Also, due to demographic change, especially in the industrial countries, the personal health care of old people is of great importance for prevention and rehabilitation. Continuous monitoring of vital parameters is essential for that aim. By long term as well as by emergency, a monitoring without interruption is crucial for the diagnosis of the case under consideration. In many cases, a motion artifact caused by patient as well as by physical motion of body parts or by the surrounding may have the same range of the signal frequency. It is difficult to filter noise from these signals, and errors resulting from filtering can distort them. Pulse oximeter for measuring oxygen saturation $\left(\mathrm{S}_{\mathrm{P}} \mathrm{O}_{2}\right)$ using more than one PPG signal is a valuable device for monitoring patients in critical conditions. PPG and the derived oxygen saturation are susceptible for motion artifact.

Pulse oximetry sensors use two Light Emitting Diodes (LEDs) which emit red and infrared light that shine through a reasonably translucent part of the patient's body. In pulse 
oximetry, it is called red light to the light band whose wavelength is comprised between 600-750 nm, while infrared light's wavelength varies between 850 and $1000 \mathrm{~nm}$. These two wavelengths values are chosen because light absorption coefficient varies with the oxygen concentration of in both the red and the infrared light. Figure 3 shows the two principles of pulse oximetry: transmission and reflection pulse oximetry. By transmission Pulse oximetry, the light sensitive photodetector (Photodiode PD), which acts as a receiver picking up the light that passes through the measuring site, is opposite to the light emitter (light emitting diode LED). By reflection pulse oximetry the PD and the LED`s lie at the same side of the irradiated body portion.

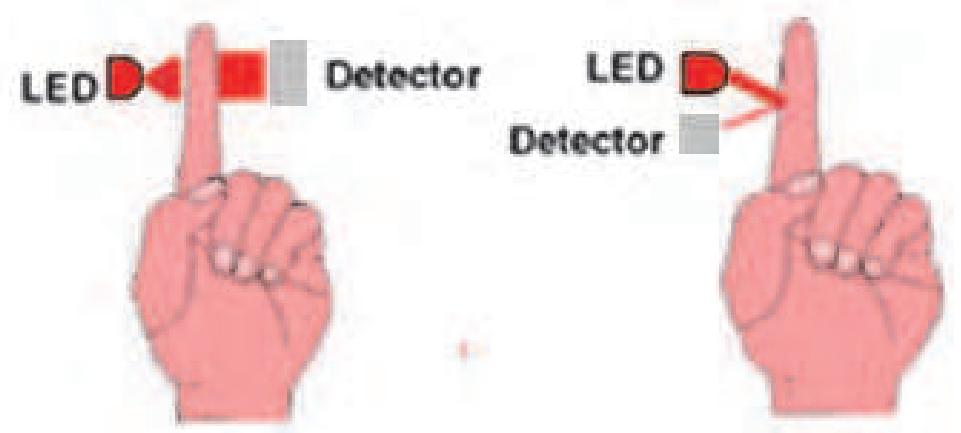

Fig. 3. Operation of the pulse oximeter sensor, left: transmission pulse oximetry, right: reflection pulse oximetry

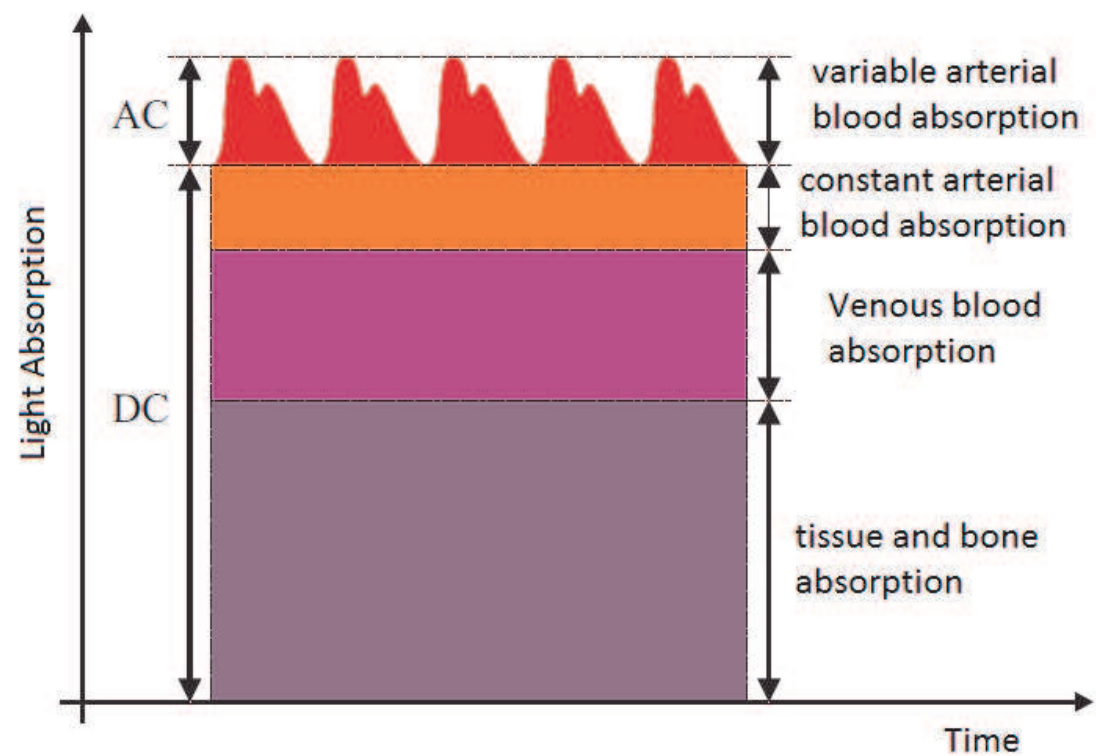

Fig. 4. Light absorption by different tissue, at the top we see the plethysmogram generated by the arterial pulsationen 
Pulse oximeter works according to two physical principles: first, the presence of a pulse wave generated by changes in blood volume (plethysmography) in the arteries and capillaries (Figure 4) and second, the fact that oxyhemoglobin $(\mathrm{O} 2 \mathrm{Hb})$ and reduced hemoglobin $(\mathrm{Hb})$ have different absorption spectra (spectroscopy). Oxygenated hemoglobin absorbs more infrared light and allows more red light to pass through. Deoxygenated (or reduced) hemoglobin absorbs more red light and allows more infrared light to pass through.

\section{Adaptive filtering of photoplethysmogram}

We emphasize heir on the use of the adaptive filter by PPG, because of the importance of this signal by detecting further parameters like pulse transit time (PPT); blood pressure monitoring, Pulse rate variability and the application of it for the risk estimation and diagnosis of cardiovascular diseases. Also the non-invasive calculation of concentration, fractional oxygen saturation and further blood components like glucose may require also the PPG signal analyzing. AC component of PPG signal caries important information for diagnosis, but it may be affected by noise, which is sharing the same bandwidth. An important application for the PPG is the calculation of oxygen saturation in emergency and in intensive care, where the oxygen supplement of tissue has to be measured continuously. The problem will be greater for example by detecting the PPG by low perfusion for the monitoring of oxygen saturation, where a low signal to noise ratio is the result. An adaptive filter will be the solution for this problem. Conventional filtering cannot be applied to eliminate those types of artifacts because signal and artifacts have overlapping spectra. For long term monitoring an adaptive filter is essential [Com 2007].

By pulse oximetry, Masimo adaptive filter is well known to the people working in this area. The principle is easy and shortly described here: all detected samples of PPG`s by red and infrared causing oxygen saturation below a certain value (e.g. $80 \%$ ) are coming from venous blood signals caused by motion artifact and has to be filtered. All signals causing saturation higher than a threshold value (e.g. 90\%) are the arterial signal. An intelligent algorithm is designed according to this principle for the robust detection of oxygen saturation. By using one PPG signal we cannot apply this algorithm. We used another algorithm by Filtering and generation of reference noise depending on the detected signal.

Motion artefacts are one of the most important handicaps of photopletysmography and pulse oximetry, as they suppose a big limitation and often become an insurmountable obstacle on the utilization of this technology, since they are quite hard to cancel mainly due to spectral characteristics of both, pulse signals and motion artifacts. In order to improve the quality of Photoplethysmograms and pulse oximetry, some signal processing must be implemented. Our research proposes, as viable solution, an Adaptive Filter in Noise Cancellation configuration, working with a Least Mean Square Algorithm. At the end of the system, we have carried out a reconstruction of the Photoplethysmogram and the signal that we recover has a high enough quality for measuring fractional oxygen saturation of hemoglobin in blood and for further diagnosis purposes.

An Adaptive Noise Cancellation (ANC) System has two inputs. This fact can be seen in the Figure 5 presented below, more specifically in the diagram on the top. One is the Input Signal, i.e., the signal corrupted by noise, coming from the sensor output, and the other one is the Noise Reference, coming from the Synthesizer output. Both, the graphic of the Input Signal and the generated plot of the Noise Reference appear in the Front Panel of the corresponding LabVIEW program. Given that the Least Mean Square Algorithm provides 
adaptive filtering, the Noise Reference is adjusted to the real noise measured with the sensor and, as a result, the output, Filtered Signal, naturally will be the filtered signal. In the diagram below from the Figure 5 the main blocks of the Least Mean Square Algorithm (LMS) implementation are presented. It is worth mentioning the fact that this algorithm is recursive: the weights of the filter are calculated recursively to minimize the Mean Square Error [Abdallah].

\section{Adaptive Noise Cancellation}



Fig. 5. Block diagram of the Adaptive Noise Cancellation (ANC)

\section{Method and results by adaptive filtering of photoplethysmogram}

Adaptive filters have been used to enable the measurement of photoplethysmogram PPG under conditions, where movement of the body parts where the sensor is applied causes a high noise to the signal. In this adaptive filter a noise reference and a signal reference are used. We use the least mean square (LMS) method to extract the actual signal from the noisy one.

For the first approximation to generate the reference signal a lowpass filter is used. Using the resulting signal from this lowpass, an appropriate reference signal is generated. This reference signal is in turn subtracted from the detected signal to generate a noise signal. The generated noise signal is modified to synthesize the noise reference signal. The synthesized reference noise is adjusted by the adaptive algorithm to the real one contained in the measurement, and then subtracted from the detected noisy signal. The resulting signal is modified to fulfill certain requirements (Figure 6)

The applied method discussed above can be used for the detection of a photopletysmogram signal without the need for further signals of the same type or requiring a further sensor. Figure 7 shows an example of the results obtained using this method. The algorithm was tested for the calculation of oxygen saturation and accurate results are delivered under artificial motion artifacts. 


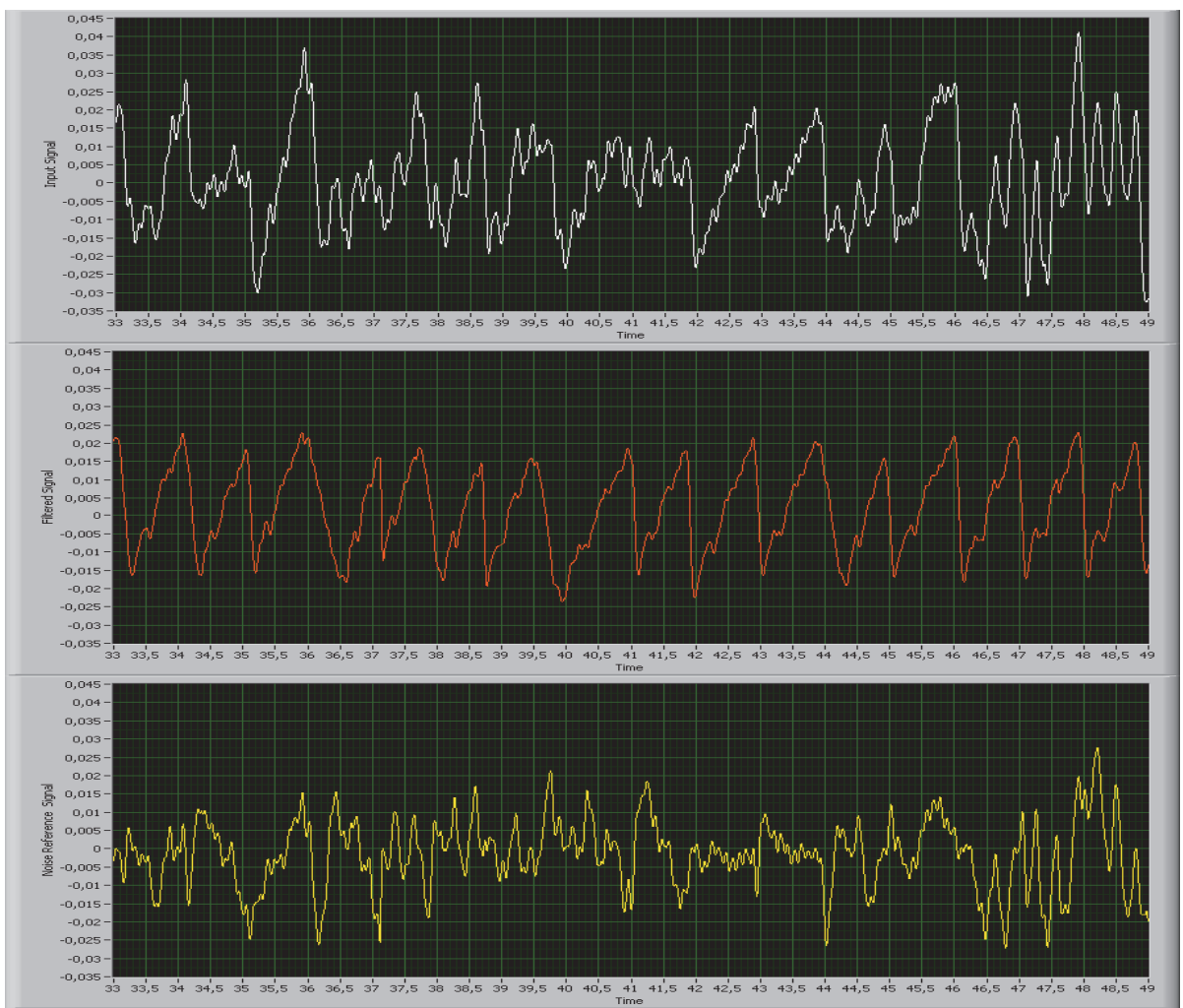

Fig. 6. Detected signal (top), generated reference signal (middle) and generated reference noise for PPG filtering

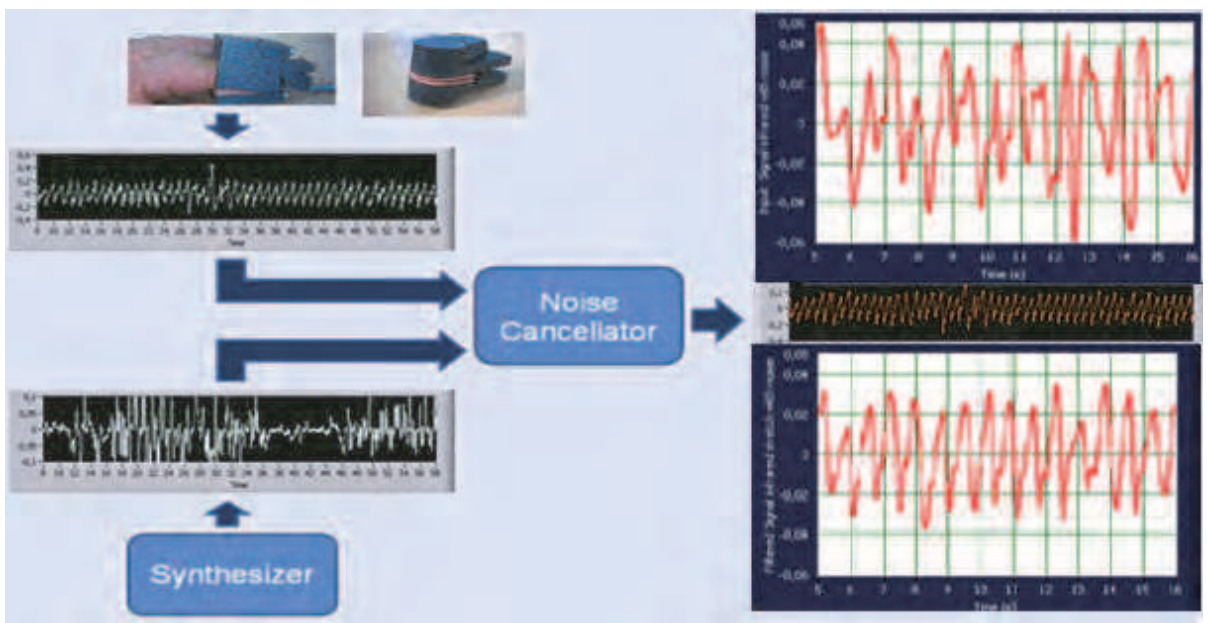

Fig. 7. Schematic of the PPG filtering 
Each measurement from the applied PHM sensor contains seven signals of LEDs having different wavelengths. Besides, a LED (which is off) acts as zero reference level. Since we need two of them, first we have to separate them. Once these signals are presented separately, we select the two of them that have been measured with the proper wavelengths value for the calculation of oxygen saturation (LED having the wavelength $970 \mathrm{~nm}$, representing infrared light and a LED having the wavelength $660 \mathrm{~nm}$, representing red light). Then they are already adapted for being filtered by our system, which remove the motion artifact from them. Finally, the filtered signals obtained after the program execution can already be used to compute ratios regarding the $\mathrm{SpO} 2$, such as the so-called $\Omega$ ratio:

$$
\Omega=\frac{\ln \frac{I\left(\lambda_{1}, t_{1}\right)}{I\left(\lambda_{1}, t_{2}\right)}}{\ln \frac{I\left(\lambda_{2}, t_{1}\right)}{I\left(\lambda_{2}, t_{2}\right)}}
$$

Where:

$I\left(\lambda_{1}, t_{1}\right), I\left(\lambda_{1}, t_{2}\right), I\left(\lambda_{2}, t_{1}\right)$ and $I\left(\lambda_{2}, t_{2}\right)$ are the light intensities measured at the instants $t_{1}, t_{2}$ with the wavelengths $\lambda_{1}, \lambda_{2}$ respectively.

As results, examples of each step of the process described here are presented. First of all, examples of the appearance of PHM measurements (and therefore, multiwavelength measurements) are shown, both the whole measurement and a zoom of it (figure 8).
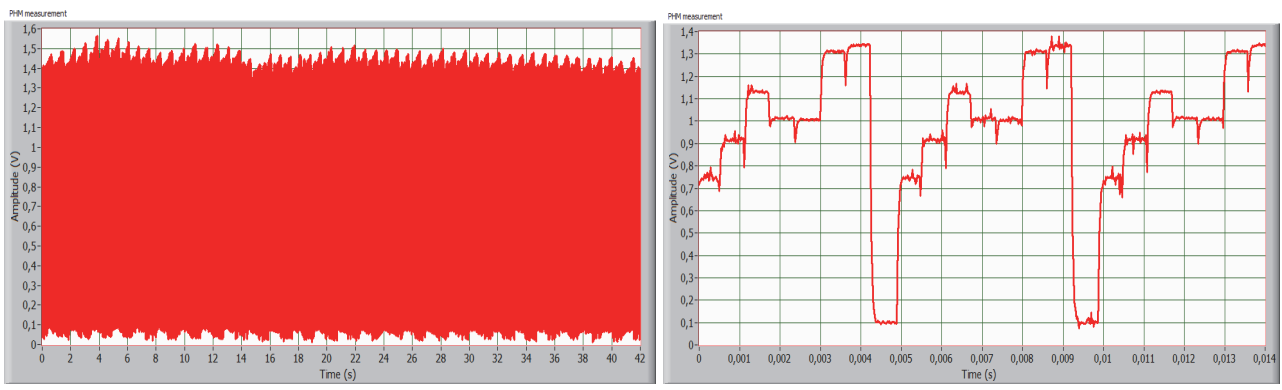

Fig. 8. Measurements of photoplethysmogram signals of PHM (right) and a zoom of it (left)

Next, the output given by the recovery of each signal is also presented. To demonstrate the ability of the system presented here to make possible a precise enough computation of the $\mathrm{SpO} 2$, we have calculated the value of the above-named $\Omega$ ratio for several measurements. In order to make sure that the adaptive filter works well enough to get accurate $\mathrm{SpO} 2$ readings, the main goals are: first, to prove that the ratios obtained are included in an acceptable range (bearing in mind that the values of this ratio allow us to estimate the calibration that has to be applied later to the exact calculation of the SpO2). Next, it must be proved that the values for the ratio when the signal is affected by motion artifacts keep quite unchanging compared to those derived from the same signals without motion artifacts [Figure 10]. The pulse amplitudes of the red and infrared signals are detected by the pulse oximeter and measured to produce a certain ratio value, which is intrinsically related to the functional oxygen saturation of (SpO2).

The signals shown in Figure 8 are measured by a Pulshemometer (PHM) sensor for the aim of calculation of concentration and fractional oxygen saturation $\mathrm{SaO} 2$, which based on the 
Principle of plethysmography (here volume change of arterial blood due to pulsation generated from the heart) and optical spectroscopy. Also by our Project for the non-invasive monitoring of glucose concentration in blood an adaptive filter for this aim is essential. For in vivo measurement of blood components, the adaptive filter is necessary to get rid of the noise and disturbances to the signal without any distortion of the detected useful signal that may cause erroneous additive signals or that may reduce the information contents in the detected signal. The Pulshemometer PHM sensor with a compact hardware circuit for driving the LED's and programmable digital potentiometer for adaptive programmable gain amplification is shown in Figure 9.



Fig. 9. Pulshemometer PHM sensor for hemoglobin concentration and fractional and fractional

Seven separated filtered PHM signals for and fractional oxygen saturation measurement von PHM are shown in Figure 11 after normalization. For this sensor an adaptive filter is essential for reliable and high accuracy results.

Wavelet transformation in combination with fuzzy and neuronal Networks (in some cases cascaded) adaptive filtering is applied by different research groups. An energy ratio-based method and a wavelet-based cascaded adaptive filter (CAF) can be applied for detecting and removing baseline drift from pulse waveforms. This CAF outperforms traditional filters both in removing baseline drift and in preserving the diagnostic information of pulse waveforms [Lisheng]. Daubechies wavelet adaptive filter based on Adaptive Linear Neuron networks is used to extract the signal of the pulse wave. Wavelet transform is a powerful tool to disclose transient information in signals. The wavelet used is adaptive because the parameters are variable, and the neural network based adaptive matched filtering has the capability to "learn" and to become time-varying. So this filter estimates the deterministic signal and removes the uncorrelated noises with the deterministic signal. This filter is found to be very effective in detection of symptoms from pulsatile part of the entire optical signal [Xiaoxia]. Fuzzy logic and Neuro-fuzzy can be used by adaptive filtering.

The method that has to be applied depends on the sensor applications and the case under consideration, because intensive computation time, a high speed processor and a large saving space may be needed, which may cause a delay time that disables an online monitoring. In applications by multi-monitoring it will be possible to use other detected signals for the purpose of filtering of a certain signal as will be discussed on the following section. 



$\mathrm{t}_{0}$ and $\mathrm{t}_{1}$ delimit a denoised

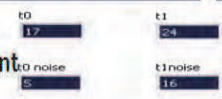

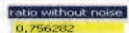

$t_{\text {Onoise }}$ and $t_{1 \text { noise }}$ delimit a noisy interval of the PHM measurementionose
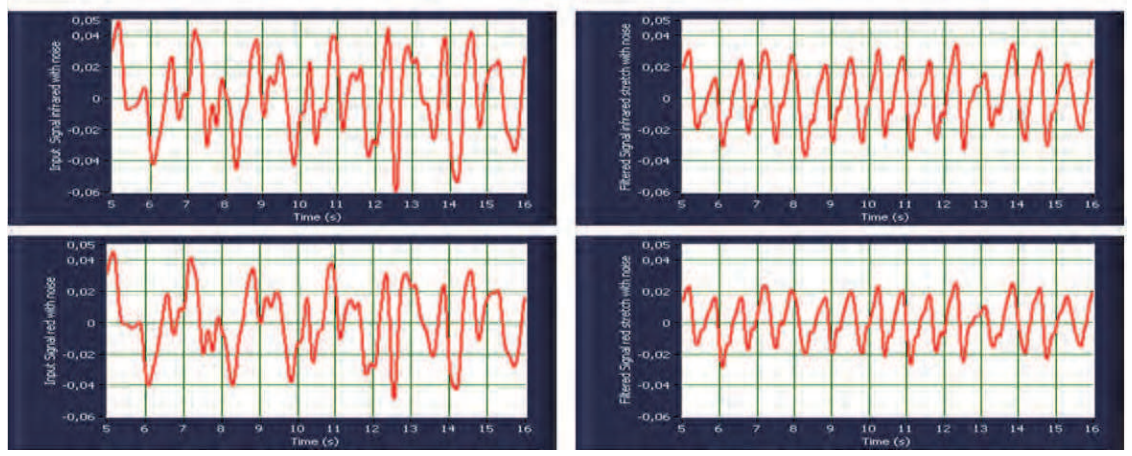

Fig. 10. LabVIEW printing for filtering a PHM measurement and computing $\Omega$

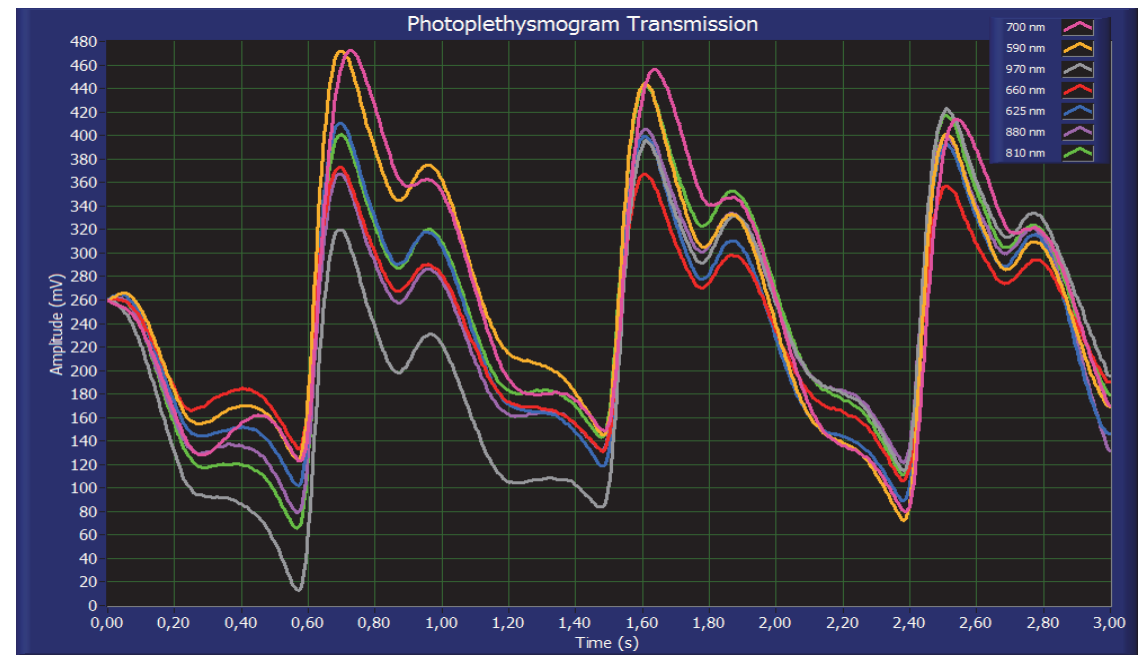

Fig. 11. LabVIEW printing for 7 filtered PHM signals for and fractional oxygen saturation measurement 


\section{Application of further signals for adaptive filter of photplethysmogram}

In one method is to use a simple acceleration sensor of a piezoelectric element [Lit. Han2009]. In a current work we use also a method based on adaptive filtering by taking the advantage of piezoelectric sensor signal to get information about the desired signal or motion to estimate the motion artifact noise or to generate the reference signal in order to get the filtered one. Hence the piezoelectric signal describes the velocity in the blood volume change; numerical integration of the piezoelectric element signal may be helpful by synthesizing of reference signal or the noise that has to be subtracted from the noisy detected signal by generating artificial motion artifacts after signal processing. Figure 12 shows the simultaneously detected optical signal detected on fingertip and a piezoelectric signal detected on the wrist with artificial motion artifact in the time period between 11 and 15 second. The piezoelectric signal is still clean and can be used for the generating of the optical signal.

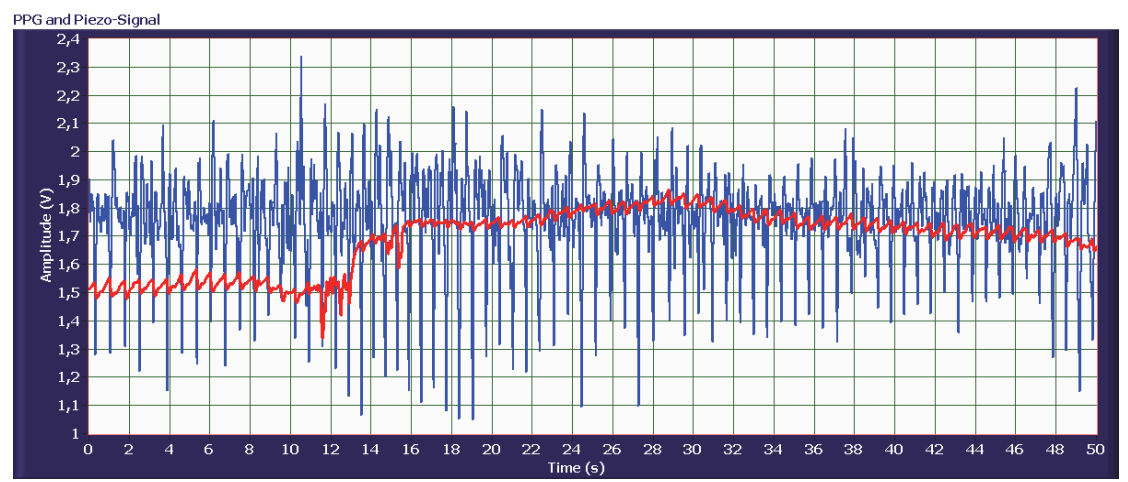

Fig. 12. PPG and piezosignal by motion artifact

Figure 13 shows the simultaneously detected optical signal detected on fingertip and a piezoelectrical signal in the case of extrasystole also detected on the wrist without motion artifact.

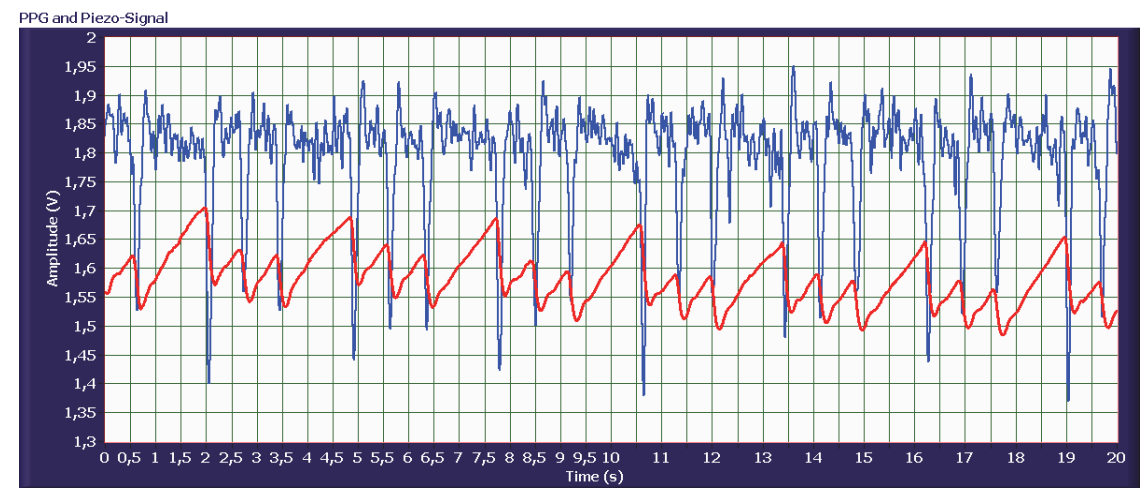

Fig. 13. PPG and piezosignal by extrasystole

Figure 14 shows the simultaneously detected optical signal detected on fingertip and the piezoelectrical signal detected on the wrist with artificial motion artifact. The piezosignal is still good enough for the generating of the PPG signal. 


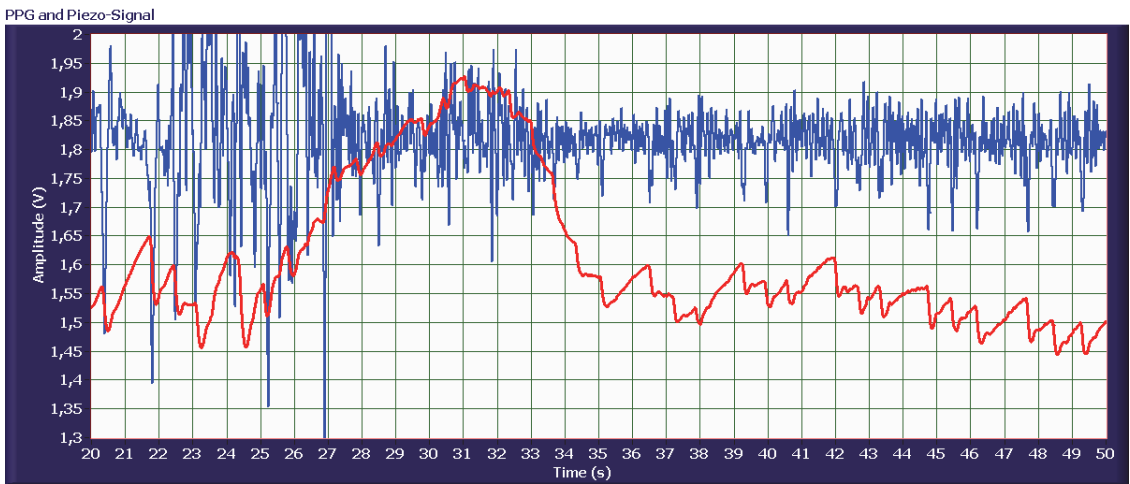

Fig. 14. PPG and piezosignal by extrasystole under motion artifact

The piezoelectric sensor may be also used for the measurement of other physiological parameters when PPG or ECG is also available. Pulse transit time PTT, which has shown its potential in cardiovascular studies, can be calculated from ECG and piezoelectric signal detected on the radial artery instead of PPG signal by low perfusion [Abdiel]. Under normal conditions the PTT can be calculated using a simple and compact piezoelectric sensor and a PPG one or by using only two simple, compact, low power piezoelectric elements. Piezoelectric elements have also different applications in biomedical engineering as sensors and actuators [Fannin].

Also a light source having the wave length around $590 \mathrm{~nm}$ can be used to generate signals that are larger than that of other wavelengths due to the high light absorption of hemoglobin at this wavelength.

Motion artifacts effects on the signal quality of photoplethysmographic signals are also discussed by different research groups. Using 3-D acceleration sensors are applied and showed very good results for adaptive filtering. The focus by [Volmer] lies on a performance estimation for a reconstruction method based on adaptive filtering with help of acceleration signals acquired at the fingertip. The acceleration in the direction of finger is an optimal setup for a continuous long-term application with low cost and low calculation complexity.

\section{Adaptive filtering by further biosignals}

The previous discussed method for the removal of the motion artifact from a PPG signal can be applied by other biosignals like ECG or EEG. We discussed in this chapter briefly the adaptive filtering by other biosignals described by different authors as examples to emphasize on the importance of adaptive filtering. Figure 15 shows a 3-chanel ECG, subjected at the end portion to motion artifact. Low signal to noise ratio can make the task of an adaptive filter more difficult.

An automated system for efficient detection of brain tumors in EEG signals using artificial neural networks (ANNs) is described by Murugesan and Sukanesh [Murugesan]. Generally, the EEG signals are bound to contain an assortment of artifacts from both subject and equipment interferences along with essential information regarding abnormalities and brain activity. Adaptive filtering has to be applied to remove the artifacts present in the EEG signal. 


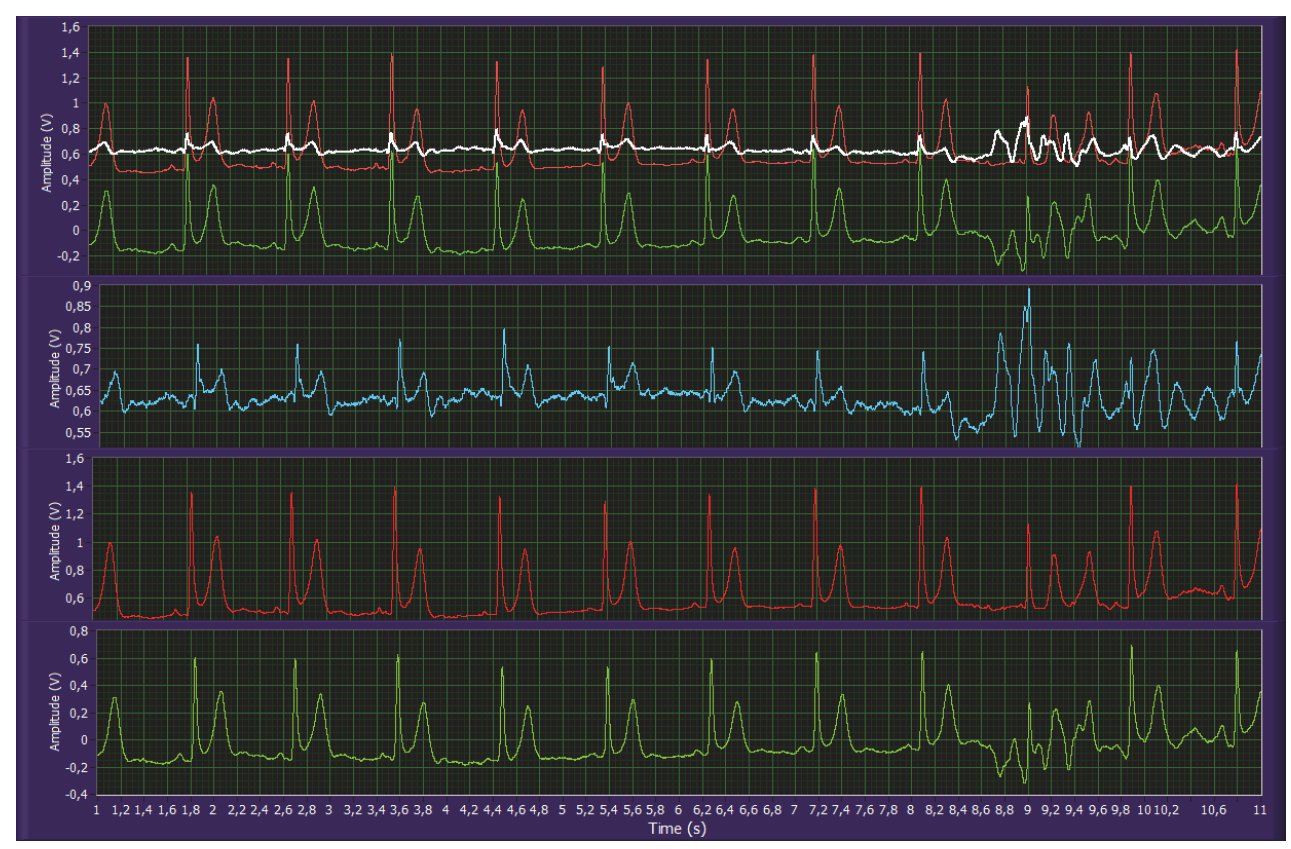

Fig. 15. ECG with motion artifact by the last three seconds

Artifacts in EEG records are caused by various factors, like line interference, EOG and ECG. These noise sources increase the difficulty in analyzing the EEG and to obtaining clinical information. For this reason, it is necessary to design specific filters to decrease such artifacts in EEG. A cascade of three adaptive filters based on a least mean squares (LMS) algorithm will be helpful. The first one eliminates line interference, the second adaptive filter removes the ECG artifacts and the last one cancels EOG spikes [Correa].

The MEG signal can also be used to control rehabilitation systems like prostheses and artificial neuromuscular electrical stimulation toward restoring movement to spinal cord injured patients. These mobile systems are usually used in different environments and thus are being exposed to different noise levels with characteristics not completely known. Different techniques for noise reduction have been compared for that aim: wavelet transform (WT), adaptive digital filters, and non-adaptive digital filters [Ortolan].

By non-invasive hemodynamic measurements like stroke volume and cardiac output, impedance cardiography (ICG) can be applied for continuously measurements. The impedance cardiography is designed for assessment and management of congestive heart failure, hypertension, and pacemaker patients. It is also appropriate for select ICU patients. The ICG waveform is generated by thoracic electrical bioimpedance (TEB) technology, which measures the level of change in impedance in the thoracic fluid [Philips]. Adaptive Filtering is in this case essential for reliable measurement at different conditions. Impedance plethysmography is a well known and extensively used noninvasive method for physical parameters monitoring. A major problem with this technique is its sensitivity to body movement. More than one frequency impedance plethysmograph can be used to measure different body parts movements. Adaptive filtering is heir very essential to detect and reduce motion artifacts [Rosell]. Wavelet based cancellation of artifacts in impedance cardiography is discussed by Pandey [Pandey]. 
Phonocardiography, Ballistocardiography may be also important for the extracting more information of the cardiovascular system as well as the low frequency signals caused by sympathic and parasympathic autonomic nervous system for the diagnostic of cardiovascular diseases and its autoregulations [Xinsheng].

Contactless measurement for physiological parameter like ECG, EEG [Oehler] and respiration rate is research area by different groups, where adaptive filtering is of great importance. Cardiac pulse based on the information contained in the thermal signal emitted from major superficial vessels is discussed by Pavlidis et. al. [Garbey]. Noncontact monitoring of breathing function using infrared imaging is also discussed by R. Murthy and I. Pavlidis.

At the end of this chapter we want only to indicate that by image processing in biomedical engineering the adaptive filtering is very essential and discussed in many literatures [Sudha]. In summary for both, long-term and short-term monitoring (for example in case of an emergency or for prevention) of biosignals, the use of an adaptive filter is essential for reliable results.

\section{Conclusions}

The motion artifact caused by subject motion as well as by physical motion of body parts has a varying frequency which may lies in the same range of the signal frequency. It is difficult to filter noise from these signals using traditional filters, and errors resulting from filtering can distort them and physicians may misled by these noisy signals and this may make the diagnosis not possible or an erroneous diagnosis is the result. By intensive care and emergency the continuous supplement of tissue with the oxygen is crucial (especially brain tissue, where after few minutes oxygen deficiency causes irreversible damage of tissue). The calculation of oxygen saturation by pulse oximetry is based on photoplethysmography and photospectroscopy. Hence the photplethysmogram plays a great rule by the calculation of oxygen saturation and heart rate as well as many further diagnostic parameters like stiffness, blood pressure and more cardiovascular pathologies can be calculated using other parameters like impedance cardiography and ECG. For a reliable detection of these parameters, an adaptive filtering is essential. By oxygen saturation detection, since many years an adaptive filter from the company Masimo that accepts all measurement results for the calculation of oxygen saturation above a threshold value and reject all measurements yielding results below another value, is robust and in clinical use. By our method using a tiefpass with a low cut off frequency a robust results were obtained. The potential of this method is that only one signal is required, which enables its application by compact sensors for different applications. Also a high velocity in the ascending portion of a signal followed by exponential or polynomial decay may consider as a PPG signal. In multi-monitoring or using multisensory for diagnosis, another signals can be used by adaptive filtering. As an example ECG or piezoelectric signals can be applied for the robust detection of PPG. For its simplicity and cost effectiveness as well as its quickly application without great disturbance of the patient we use piezoelectric signals. Acceleration sensors find their application by the adaptive filtering for PPG or ECG and further physiological parameters. By multisensors some signals can be used to generate the reference signal that has to be extracted from the measured signal or the reference noise that has to be removed from the measured data. Kalman Filter, Neuronal Fuzzy with optimization methods like swarm algorithms and Wavelet Transformation may be in some cases a good choice for 
adaptive filtering of biosignals. The method which has to be applied depends on the case under consideration and the availability of other sensors. For emergency, intensive care, home care and long term monitoring and over all, where non-invasive measurement are applied, the use of adaptive filter is of a great importance and in many cases is compulsory to get the required results. It will also radically reduce the disturbances (alarm) for patient and medical care stuff, reduce costs and enhance the medical systems.

\section{References}

Abdallah, O.; Piera Tarazona, A., Martínez Roca, T., Boutahir, H., Abo Alam, K. \& Bolz, A. (2006). Photoplethysmogram Signal Conditioning by Monitoring of Oxygen Saturation and Diagnostic of Cardiovascular Diseases, 4th European Congress for Medical and Biological Engineering, ISBN 978-3540892076, pp. (303-306), Antwerp, September 2008,

Abdiel Foo Jong Yong \& Sing Lim Chu. (2006). Pulse Transit Time based on Piezoelectric Technique at the redial Artery. Journal of clinical monitoring and computing, (May 2006) Vol. 20, Nr. 3, pp. 185-192

Abicht Jan-Michael. (2003) Computerunterstuetzte Analyse photoplethysmographischer Signale, Dissertation zum Erwerb des Doktorgrades der Medizin an der Medizinischen Fakultaet der Ludwig-Maximilians Universitaet zu München, October 2003, available from: http://edoc.ub.uni-muenchen.de/1793/1/Abicht_Jan_Michael.pdf

Allen John. (2007) Photoplethysmography and its application in clinical physiological Measurement, Physiological Measurement 28, , (February 2007), pp. R1-R39

Comtois, G.; Mendelson, Y., Ramuka, P. (2007). A Comparative Evaluation of Adaptive Noise Cancellation Algorithms for Minimizing Motion Artifacts in a Forehead-Mounted Wearable Pulse Oximeter, Conf proceeding IEEE Eng Medicine Biology Soc EMBS, ISBN: 978-1-4244-0787-3, pp. 1528 - 1531, Lyon, August 2007

Fannin Christopher A. (1997). Design of an Analog Adaptive Piezoelectric Sensoriactuator, Thesis submitted to the Faculty of the Virginia Polytechnic Institute and State University in partial fulfillment of the requirements for the degree of MASTER OF SCIENCE, 1997

From : http:/ / schoöar.lib.vt.edu/thesis/etd-8897-171952/unrestrictd/Cfannin.pdf

Garbey Marc Sun Nanfei, Merla Arcangelo, \& Pavlidis Ioannis. (2007). Contact-Free Measurement of Cardiac Pulse Based on the Analysis of Thermal Imagery, IEEE Transactions On Biomedical Engineering, Vol. 54, No. 8, August 2007, pp. 1418-1426

Han D. K., Hong J. H., Shin J. Y. \& Lee T. S. (2009). Accelerometer based motion noise analysis of ECG signal, World Congress on Medical Physics and Biomedical Engineering, , IFMBE Proceedings, Vol. 25/5, pp. 198-201, Munich, Germany, September 2009

Lee, Ju-Won and Lee, Gun-Ki (2005) Design of an Adaptive Filter with a Dynamic Structure for ECG Signal Processing, International Journal of Control, Automation, and Systems, vol. 3, no. 1, (March 2005), pp. 137-142,

Lisheng, X., David Z., \& Kuanquan W. (2005). Wavelet-Based Cascaded Adaptive Filter for Removing Baseline Drift in Pulse Waveforms, IEEE Transactions on Biomedical Engineering, Vol. 52, No. 11, (November 2005), pp. 1973-1975, ISSN 0018-9294

Murugesan M. \& Sukanesh R. (2009) Towards Detection of Brain Tumor in Electroencephalogram Signals Using Support Vector Machines, International Journal of Computer Theory and Engineering, Vol. 1, No. 5, (December, 2009), pp. 1793-8201

Murugesan, M. \& Sukanesh, R. (2009). Automated Detection of Brain Tumor in EEG Signals Using Artificial Neural Networks, Int. Conf. on Advances in Computing, Control, and Telecommunication Technologies, pp. 284 - 288, Trivandrum, India, December 2009 
Oehler Martin Johannes (2009) Kapazitive Elektroden zur Messung bioelektrischer Signale, Technischen Universitaet Carolo-Wilhelmina zu Braunschweig, Dissertation 2009

Available from: http://rzbl04.biblio.etc.tubs.de:8080/docportal/receiv/DocPortal_document_00031116

Ortolan, RL., Mori, RN. Pereira, RR., Cabral, CM., Pereira, JC. \& Cliquet, AJ. (2003) Evaluation of adaptive/nonadaptive filtering and wavelet transform techniques for noise reduction in EMG mobile acquisition equipment, Neural Systems and Rehabilitation Engineering, IEEE Transactions Vol. 11, No. 1, (March 2003) pp. 60 - 69, ISSN 1534-4320

Pandey Vinod, K. \& Pandey Prem, C. (2007). Wavelet based cancellation of respiratory artifacts in impedance cardiography, IEEE Intl. Conf. on Digital Signal Processing, Cardiff, Wales, UK, July 2007

Philips Healthcare: ICG Impedance Cardiography, Non-invasive hemodynamic measurements, http://www.healthcare.philips.com/main/products/patient_monitoring/pr oducts/icg/

Prasad D.V. \& Swarnalatha R. (2009) A New Method of Extraction of FECG from Abdominal Signal, Int. Conf. On Biomedical Engineering, IFMBE Proceedings, Vol. 23, pp. 98-100, Singapore, December 2008

Rasheed Tahir, Ho In Myung, Lee, Young-Koo, Lee Sungyoung, Lee Soo Yeol \& Kim TaeSeong (2006). Constrained ICA Based Ballistocardiogram and Electro-Oculogram Artifacts Removal from Visual Evoked Potential, EEG Signals Measured Inside MRI, Lecture Notes in Computer Science, Vol. 4232, 2006, pp. 1088-1097,

Rik Vullings, Chris Peters, Massimo Mischi, Rob Sluijter, Guid Oei, \& Jan Bergmans (2007) Artifact reduction in maternal abdominal ECG recordings for fetal ECG estimation, Proceedings of the 29th Annual International Conference of the IEEE EMBS, Lyon, France, August 2007

Rosell, Javier., Cohen Kevin P. \& Webster John G. (1995) Reduction of motion artifacts using a two-frequency impedance plethysmograph and adaptive filtering, IEEE Transactions On Biomedical, Engineering, Vol. 42, No. 10, (October 1995), pp.1044-148, ISSN 00189294

Sudha S., Suresh G. R. \& Sukanesh R. (2009) Speckle Noise Reduction in Ultrasound Images by Wavelet Thresholding based on Weighted Variance, International Journal of Computer Theory and Engineering, Vol. 1, No. 1, April 2009, pp. 1793-8201,

Rasheed T., Young-Koo L., Soo LY. and Kim TS. (2009). Attenuation of artifacts in EEG signals measured inside an MRI scanner using constrained independent component analysis, Physiol. Meas., Vol. 30, No. 4, April 2009, pp. 387-404

Volmer Achim, Orglmeister Reinhold \& Feese Sebastian. (2010). Motion Artifact Compensation for Photoplethysmographic Signals by Help of Adaptive Noise Cancelation Motion, Automatisierungstechnik, Vol. 58, No. 5, May 2010, pp. 269-276

Xiaoxia Li, Gang Li, Ling Lin, Yuliang Liu, Yan Wang \& Yunfeng Zhang. (2004). Application of a Wavelet Adaptive Filter Based on Neural Network to Minimize Distortion of the Pulsatile Spectrum, Advances in Neural Networks Lecture Notes in Computer Science, Vol. 3174, 2004, pp. 279-301, ISNN 2004

Xinsheng Yu, Don Dent \& Colin Osborn. (1996). Classification of Ballistocardiography using Wavelet Transform and Neural Networks, Annual International Conference of the IEEE Engineering in Medicine and Biology Society, Vol. 3, October 1996, pp. 937 - 938, ISBN 07803-38111

Yan, YS., Poon CC., \& Zhang, YT. (2005). Reduction of motion artifact in pulse oximetry by smoothed pseudo Wigner-Ville distribution, Journal of NeuroEngineering and Rehabilitation, 2 : 3, March 2005, 


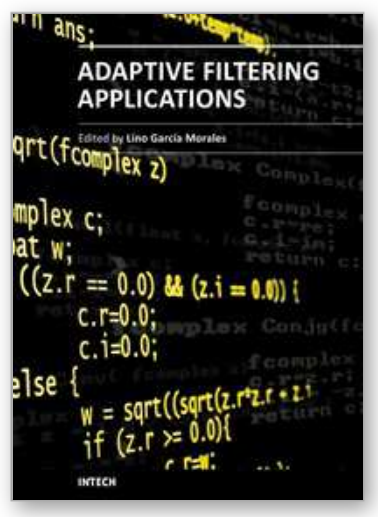

\author{
Adaptive Filtering Applications \\ Edited by Dr Lino Garcia
}

ISBN 978-953-307-306-4

Hard cover, 400 pages

Publisher InTech

Published online 24, June, 2011

Published in print edition June, 2011

Adaptive filtering is useful in any application where the signals or the modeled system vary over time. The configuration of the system and, in particular, the position where the adaptive processor is placed generate different areas or application fields such as: prediction, system identification and modeling, equalization, cancellation of interference, etc. which are very important in many disciplines such as control systems, communications, signal processing, acoustics, voice, sound and image, etc. The book consists of noise and echo cancellation, medical applications, communications systems and others hardly joined by their heterogeneity. Each application is a case study with rigor that shows weakness/strength of the method used, assesses its suitability and suggests new forms and areas of use. The problems are becoming increasingly complex and applications must be adapted to solve them. The adaptive filters have proven to be useful in these environments of multiple input/output, variant-time behaviors, and long and complex transfer functions effectively, but fundamentally they still have to evolve. This book is a demonstration of this and a small illustration of everything that is to come.

\title{
How to reference
}

In order to correctly reference this scholarly work, feel free to copy and paste the following:

Omar Abdallah and Armin Bolz (2011). Adaptive Filtering by Non-Invasive Vital Signals Monitoring and Diseases Diagnosis, Adaptive Filtering Applications, Dr Lino Garcia (Ed.), ISBN: 978-953-307-306-4, InTech, Available from: http://www.intechopen.com/books/adaptive-filtering-applications/adaptive-filtering-by-noninvasive-vital-signals-monitoring-and-diseases-diagnosis

\section{INTECH}

open science | open minds

\section{InTech Europe}

University Campus STeP Ri

Slavka Krautzeka 83/A

51000 Rijeka, Croatia

Phone: +385 (51) 770447

Fax: +385 (51) 686166

www.intechopen.com

\section{InTech China}

Unit 405, Office Block, Hotel Equatorial Shanghai

No.65, Yan An Road (West), Shanghai, 200040, China

中国上海市延安西路65号上海国际贵都大饭店办公楼405单元

Phone: +86-21-62489820

Fax: +86-21-62489821 
(C) 2011 The Author(s). Licensee IntechOpen. This chapter is distributed under the terms of the Creative Commons Attribution-NonCommercialShareAlike-3.0 License, which permits use, distribution and reproduction for non-commercial purposes, provided the original is properly cited and derivative works building on this content are distributed under the same license. 